\title{
RECIEN
}

Revista Cientifica de Enfermeria

\section{HÁBITOS NUTRICIONALES EN DEPENDIENTES A LA HEROÍNA}

NUTRITIONAL HABITS IN HEROIN ADDICTS

Quintero-Febles J*

Suárez-Sánchez JJ**

*Unidad de Desintoxicación Hospitalaria del Hospital Universitario de Canarias. Servicio Canario de Salud.

Departamento de Enfermería, Escuela Universitaria de Enfermería y Fisioterapia de la Universidad de La Laguna

** Equipo Atención Primaria, Zona Básica de Salud de Ingenio. Servicio Canario de Salud.

Departamento de Enfermería, Escuela Universitaria de Enfermería y Fisioterapia de la Universidad de La Laguna. 


\section{RESUMEN:}

\section{Título:}

Hábitos nutricionales en dependientes a la heroína

\section{Objetivos:}

Analizar el estado nutricional y evaluar el consumo alimentario, conocimientos y actitudes en relación con la alimentación en un grupo de adictos a la heroína.

\section{Método:}

Se trata de un estudio observacional analítico basado en registro de valores antropométricos y entrevista alimentaria a los pacientes que realizan un tratamiento de desintoxicación.

\section{Resultado:}

Se observa disminución significativa de la media del índice de masa corporal, horario de comidas irregular, predominio del consumo de dulces, bollería y comida rápida.

\section{Conclusiones:}

La población compuesta por individuos con dependencia a la heroína se encuentra en una situación de elevado riesgo de desarrollo de problemas relacionados con la malnutrición.

\section{Palabras clave:}

Dieta, Nutrición de Grupos Vulnerables, Conducta Adictiva, Dependencia de Heroína. 


\section{ABSTRACT:}

\section{Title:}

Nutritional habits in heroin addicts.

\section{Objectives:}

Analyze the nutritional status and evaluate food consumption, knowledge and attitudes in relation to feeding in a group of addicts to the heroine.

\section{Method:}

Record of anthropometric values and food interview to the patients who realize a treatment of detoxification.

\section{Results:}

Significant decrease of the mean body mass index, irregular meal times, dominance of sweets, pastries and fast-food consumption.

\section{Conclusions:}

The population of individuals with heroin dependence is in a situation of high risk of developing problems associated with malnutrition.

\section{Key words:}

Diet, Risk Group Nutrition, Behavior Addictive, Heroin Dependence. 


\section{INTRODUCCIÓN:}

La Unidad Desintoxicación Hospitalaria (UDH) del Hospital Universitario de Canarias atiende a la población de drogodependientes de Santa Cruz de Tenerife, siendo el único recurso de este tipo en la provincia. Esta determinante y privilegiada situación del equipo terapéutico de la UDH proporciona el marco ideal para realizar una valoración de los hábitos nutricionales y trastornos alimentarios de una población en alto riesgo. La población con problemas adictivos se encuentra en alto riesgo tanto por su característico estilo de vida como por una falta de interés por cualquier cosa ajena al consumo de drogas, descuidando su salud, higiene, nutrición e inclusive dejando de solicitar asistencia sanitaria cuando lo necesitan ${ }^{1-2}$. La drogodependencia predispone no sólo a desordenes nutricionales relacionado con una ingesta deficiente de alimentos, sino también a aquellos desordenes relacionados con el abuso de alimentos. En el caso concreto de los adictos a la heroína, la presencia de patologías orgánicas agudas, como el VIH o las hepatitis, conducen a un empeoramiento de su estado nutricional ${ }^{3}$. Resulta por tanto de gran interés, para todos los profesionales que trabajen con drogodependientes, conocer las especificidades de los adictos a la heroína en relación con la nutrición. Indudablemente los efectos causados por las diferentes drogas en el individuo siempre serán perjudiciales, ya sea por los propios efectos de la sustancia o por las implicaciones sociales y culturales que su consumo implica. Diversos estudios experimentales ${ }^{4}$ demuestran una implicación del sistema opioide endógeno en la regulación del apetito. Los opiáceos ejercen un efecto estimulante en el apetito de los dulces, la glucosa aumenta la producción de opioides endógenos, que normalmente estará deprimida en los adictos a los opiáceos. Los niveles de glucosa bajos en adictos a estas sustancias parecen explicar la preferencia por glúcidos de rápida asimilación, con el fin de compensar el déficit de su ingesta energética.

Hay que tener en cuenta que la combinación de una dieta rica en azucares simples y una mala higiene bucal (por dejadez y desinterés en el cuidado personal) hacen que lo trastornos de gingivitis y caries dentales sean 
muy frecuentes en pacientes adictos a los opiáceos. Esta situación contribuye a la modificación de los hábitos dietéticos, mostrándose una tendencia hacia los alimentos de textura blanda y fácil masticación, apareciendo cuadros clínicos de malnutrición. A ello se añade un consumo excesivo de alcohol, tabaco y café que aumentan la disminución de la ingesta de alimentos y producen una mala absorción por alteraciones en la mucosa gástrica e intestinal, contribuyendo a una malnutrición ${ }^{5}$. Los déficits nutricionales son debidos tanto a un estilo de vida desordenado y a una falta de interés por la alimentación como a problemas específicos del consumo. El presente estudio realizado en la UDH supone un primer análisis de la situación actual de los opiaceodependientes en relación con sus hábitos nutricionales. Sus resultados nos permitirán dilucidar la necesidad de desarrollar estudios en profundidad y actividades de educación sanitaria.

\section{METODOLOGÍA}

Se trata de un estudio observacional analítico basado en datos de registros sobre pacientes opiaceodependientes que se encuentran realizando de forma voluntaria un tratamiento de desintoxicación hospitalaria.

\section{Población y muestra:}

Se seleccionaron a todos los pacientes adictos a la heroína que solicitaron ingreso en la UDH durante un periodo de seis meses, (febrerojulio/2010) remitidos desde las Unidades de Atención al Drogodependiente. La muestra alcanzada ha representado un total de 44 individuos, con una proporción de 9 mujeres y 35 hombres, la media de edad ha sido de 33 años.

\section{Criterios de inclusión:}

Estar realizando un tratamiento de desintoxicación hospitalaria por adicción a la heroína y aceptar libremente la participación en el estudio. 


\section{Criterios de exclusión:}

En el diseño del estudio se plantearon como criterios de exclusión la presencia de barreras idiomáticas que impidieran la realización de la entrevista y aquellos pacientes que rechazaran participar el estudio.

\section{Variables objeto de estudio:}

La valoración constó de dos partes, en una primera parte se registraron los valores antropométricos correspondientes al peso, talla, Índice de Masa Corporal (IMC), perímetro braquial, perímetro abdominal y perímetro de cadera. La segunda parte consistió en una entrevista alimentaria que a su vez se divide en tres secciones: encuesta de distribución de las comidas, encuesta de conocimientos, opiniones y actitudes y los cuestionarios de frecuencia de consumo de alimentos.

\section{Análisis estadístico:}

La comparación de los resultados obtenidos en el presente estudio, en comparación con los obtenidos por estudios realizados en la población canaria en general ${ }^{6}$, nos muestran una disminución de los parámetros antropométricos: peso, IMC, y los perímetros braquial, abdominal y de cadera. En las encuestas referentes a la distribución de las comidas, se le solicitó a cada participante que indicara los lugares y momentos en los que realizaba las ingestas. Se plantearon las preguntas cerradas, a elegir entre diversas opciones: nunca, menos de una vez al mes, menos de una semanal, una a la semana, más de una a la semana. Las respuestas indicadas por la población a estudio nos indican un horario irregular de ingestas y una disminución del número total de comidas aunque con un aumento de las comidas fuera de casa (Tabla 1). Para la evaluación de los alimentos elegidos por los encuestados se optó por los cuestionarios de frecuencia de consumo (CFC), se trata de un método barato, simple y rápido que nos da una información cualitativa del consumo de alimentos ${ }^{9}$. El consumo se refiere al habitual en el último año y las 
posibilidades de respuesta son: diaria, semanal, mensual y no consumo. Cuando analizamos los resultados y en comparación con la población canaria observamos una disminución del consumo de pescado, legumbres, frutas y hortalizas, confirmando un abuso del consumo de dulces y bollería (Tabla 2).

La valoración de los conocimientos, opiniones y actitudes es igualmente relevante ya que, la diferencia entre lo que se aconseja a la población y lo que esta consume, pudiera ser debida en parte, al desconocimiento de la misma sobre alimentación saludable y guías dietéticas ${ }^{8}$. Llamativo resulta que el $90 \%$ de la muestra afirme creer tener unos conocimientos adecuados sobre nutrición, muy por encima de la población en general $(46,7 \%)$.

\section{DISCUSIONES}

Los resultados del presente estudio nos sugieren un deficiente estado nutricional en la población con problemas adictivos relacionados con la heroína.

La significativa disminución en la media del IMC parece indicarnos que los desordenes nutricionales son más por defecto que por exceso, en consonancia con los resultados obtenidos por estudios similares ${ }^{1-2}$. En la valoración de las comidas encontramos una moderada disminución del número de las comidas, aunque no tanto como era de esperar por otros estudios ${ }^{1-2}$. En la valoración de los conocimientos y actitudes, lo que más llama la atención es la tendencia observada a sobrevalorar los propios conocimientos sobre nutrición y una desmesurada predisposición a cambiar los hábitos. Es conveniente recordar que el sujeto se encuentra inmerso en una desintoxicación y deshabituación de sus hábitos de consumo, lo que conlleva indudablemente una predisposición al cambio. En los cuestionarios de frecuencia de consumo, encontramos un perjudicial aumento en el consumo de dulces, y grasas, disminuyendo drásticamente el consumo de frutas y 
hortalizas respecto a la población canaria ${ }^{6}$, en la misma línea que estudios similares $^{1-4}$. Esta primera aproximación ha sido realizada con una muestra reducida y seleccionando sólo a los pacientes que solicitan un tratamiento de desintoxicación, por lo que sus resultados no pueden ser extrapolados al total de la población de opiaceodependientes, no obstante, ofrecen unas orientaciones, que pueden servir de base a posteriores análisis.

\section{CONCLUSIONES}

En el presente trabajo hemos encontrado, en la muestra seleccionada de pacientes adictos a la heroína, unas tendencias a un horario irregular de las comidas, predominio del uso de comida rápida y bollería, sobrevaloración de los propios conocimientos y predisposición al cambio. Todas estas orientaciones que hemos encontrado sugieren un deficiente estado nutricional de la población drogodependiente, reforzando la necesidad de realizar un estudio a fondo del estado y los hábitos nutricionales en una población de alto riesgo así como desarrollar actividades de educación para la salud alimentaria.

\section{BIBLIOGRAFIA}

1. Del Mar Verde Méndez C, Díaz-Flores JF, Sañudo RI, Rodríguez-Rodríguez E, Díaz Romero C. Hábitos alimentarios en opiaceo-dependientes incluidos en un programa de mantenimiento con metadona. Alim Nutr Salud 2002; 9 (3):61-8

2. Santolaría Fernández FJ, Gómez Sirvent JL, González Reimers CE. Batista López JN, Jorge Hernández JA, Rodríguez Moreno F, Martínez Riera A, Hernández García MT. Nutritional assessment of drug addict. Drug and Alcohol Dependence 1995; 38:11-8

3. Varela G, Marco A, Santacruz I, Ripoll S, Requejo AM. Human inmunodeficiency virus infection and nutritional status in female drug addicts undergoing detoxification: anthropometric and immunologic assessments. Am 
Clin Nutr 1997; 66: 504-8

4. Graña Gómez JL, Andreu JM, Muñoz Rivas M. Preferencia al sabor en los opiáceos. Psicothema 2005; 17:201-4

5. Lorenzo P, Leza JC, Lizasoain I. Drogodependencias. Madrid: Ed. Mc GrawInteramericaca, 1993, 498-529

6. Equipo Investigador ENCA (1997-1998). Encuesta Nutricional de Canarias. Santa Cruz de Tenerife. Servicio Canario de Salud. 1999

7. Verde Méndez $C$ del M, Díaz Flores JF, Sañudo R I, Rodríguez E M, Díaz Romero C. Área de Nutrición y Bromatología. Universidad de La Laguna. Parámetros hematológicos en pacientes opiaceodependientes. Nutr Hosp 2003; 18: 358-365

8. Serra-Majem L, Ribas L. Hábitos alimentarios y consumo de alimentos en España. Dieta mediterránea. Barcelona: Masson S.A; 1995. 303-10.

9. Gorgojo Jiménez L, Martín Moreno JM, Royo Bordonada MA (coord.) Nutrición en Salud Pública. Madrid. Instituto de Salud Carlos III. 2007. 235-55 


\section{TABLA 1}

(\% población drogodependientes / \% población canaria)

Frecuencia de comidas realizadas fuera de casa

\begin{tabular}{|l|c|c|c|c|c|}
\cline { 2 - 5 } \multicolumn{1}{c|}{} & Amigos & Restaurante & Fast-Food & Bar & Comedor \\
\hline Nunca & $50 \% / 29,2 \%$ & $10 \% / 30,7 \%$ & $30 \% / 67 \%$ & $10 \% / 77 \%$ & $90 \% / 85,3 \%$ \\
\hline Menos de una al mes & $30 \% / 18,4 \%$ & $90 \% / 21,7 \%$ & $30 \% / 6 \%$ & $20 \% / 5,1 \%$ & $0 \% / 1,1 \%$ \\
\hline Menos de una semanal & $30 \% / 29,2 \%$ & $0 \% / 33,5 \%$ & $30 \% / 16,7 \%$ & $20 \% / 10,5 \%$ & $0 \% / 1,3 \%$ \\
\hline Una a la semana & $0 \% / 13,9 \%$ & $0 \% / 8,6$ & $10 \% / 6,2 \%$ & $30 \% / 3,4 \%$ & $0 \% / 0,7 \%$ \\
\hline Más de una semanal & $0 \% / 9,42 \%$ & $0 \% / 4,6 \%$ & $0 \% / 4,4 \%$ & $20 \% / 31,5 \%$ & $10 \% / 11,6 \%$ \\
\hline
\end{tabular}

\section{TABLA 2}

(\% población drogodependientes / \% población canaria)

Frecuencia de consumo de dulces y bollería

\begin{tabular}{|l|c|c|c|c|}
\cline { 2 - 4 } \multicolumn{1}{c|}{} & Diaria & Semanal & Mensual & No consumo \\
\hline Azúcar & $90 \% / 73,4 \%$ & $10 \% / 3,4 \%$ & $0 \% / 4,5 \%$ & $0 \% / 18,9 \%$ \\
\hline Miel & $0 \% / 5,4 \%$ & $0 \% / 4,6 \%$ & $0 \% / 24,7 \%$ & $80 \% / 65,3 \%$ \\
\hline Bollería & $30 \% / 8 \%$ & $50 \% / 16 \%$ & $10 \% / 36,7 \%$ & $10 \% / 39,3 \%$ \\
\hline Dulces y pasteles & $30 \% / 4,1 \%$ & $40 \% / 12,4 \%$ & $20 \% / 50,7 \%$ & $10 \% / 32,9 \%$ \\
\hline Galletas & $40 \% / 22,1 \%$ & $40 \% / 22,9 \%$ & $10 \% / 30,9 \%$ & $10 \% / 24,2 \%$ \\
\hline Caramelos yolosinas & $30 \% / 22,1 \%$ & $30 \% / 16,8 \%$ & $10 \% / 19,8 \%$ & $30 \% / 41,3 \%$ \\
\hline
\end{tabular}

